\title{
Ebook como Tecnologia Educacional no Ensino do Gerenciamento em Segurança do Escolar com Alergia a proteína do Leite de Vaca
}

\author{
Ebook as an Educational Technology in Teaching Safety Management of Students with Allergy to \\ Cow's Milk Protein
} EI Ebook como tecnología educativa en la enseñanza de la gestión de la seguridad de los estudiantes
con alergia a la proteína de la leche de vaca

Débora Cristina Mendonça de Andrade ${ }^{1}$, Ana Karine Ramos Brum²

Como citar esse artigo. de Andrade, DCM; Brum, AKR. Ebook como Tecnologia Educacional no Ensino do Gerenciamento em Segurança do Escolar com Alergia a proteína do Leite de Vaca. Revista Pró-UniverSUS. 2019 Jul./Dez.; 10 (2): 89-92.

\section{Resumo}

Introdução: Dados da Associação Brasileira de Alergia e Imunologia afirmam que entre 6\% e 8\% das crianças têm alergias alimentares, e dentre elas a alergia a proteína do leite de vaca (APLV) é a causa mais comum, considerada atualmente um problema de saúde pública. Para criança que apresenta reações alérgicas alimentares graves o ambiente extradomiciliar, representa alto risco para desencadear anafilaxia. Ao analisar este cenário, constata-se a seriedade de ter um enfermeiro na escola. O objetivodo estudo é desenvolver uma tecnologia educacional no formato de Ebook para o ensino do gerenciamento em segurança do escolar APLV na formação profissional dos enfermeiros e profissionais de educação. Método: Estudo descritivo, exploratório, de abordagem qualitativa com desenvolvimento de pesquisa metodológica para elaboração de uma tecnologia educacional. Os participantes serão estudantes de enfermagem de uma Universidade Federal, profissionais de educação de uma escola pública e privada nos municípios de Maricá e Niterói, respectivamente. A pesquisa obteve parecer favorável pelo Comitê de Ética em Pesquisa da Universidade Federal Fluminense. Serão agendadas três oficinas, onde será aplicado um questionário semiestruturado. O tratamento e a análise dos dados serão feitos de forma descritiva, através da análise e classificação do conteúdo.Resultados esperados:Pretende-se trazer benefícios ao ensino, pesquisa e a sociedade, discutindo questões que subsidiarão a reorientação do processo de formação dosenfermeiros, engajando-os para que possam atuar conjuntamente com os profissionais da educação, a fim de alcançar a principal meta que é a qualidade do cuidado e a segurança do escolar com APLV.

Palavras-chave: Serviços de Saúde Escolar, Tecnologia Educacional, Segurança do Paciente.

\begin{abstract}
Objectives: Introduction: The Brazilian Association of Allergy and Immunology estimates that $6 \%$ to $8 \%$ of children have food allergies, and among them cow's milk protein allergy (APLV) is the most common cause, which is currently considered a public health problem. For those children with severe food allergic reactions, the outside home environment represents a high risk of triggering anaphylaxis. By analyzing this scenario, it is possible to attest how critical it is to have a nurse at school. This study aims at developing an educational technology in the format of eBook to teach nurses and education professionals safety management of students with APLV during their professional training. Method: Descriptive, exploratory study of qualitative approach with development of methodological research for the elaboration of an educational technology. Participants will be nursing students from a Federal University, education professionals from a public and private school in the municipalities of Maricá and Niterói, respectively. The research obtained a favorable assessment by the Research Ethics Committee of the Fluminense Federal University. Three workshops will be scheduled and a semi-structured questionnaire will be applied then. The treatment and analysis of the data will be done descriptively, through the analysis and classification of the content. Expected Outcomes: It is expected that this study will benefit teaching, research and society, by discussing issues that will subsidize a review of the nurses' training process, engaging them so that they can work alongside with the education professionals to reach a common goal, which is the quality of care and safety of the student with APLV.
\end{abstract}

Keywords: School Health Services, Educational Technology, Patient Safety

Afiliação dos autores: 1. Enfermeira. Mestranda do programa de Mestrado Profissional em Ensino na Saúde/MPES, UFF, RJ, Brasil. Email: deboracmandrade@yahoo.com.br ORCID: https://orcid.org/0000-0003-4865-9480

2. Enfermeira. Pós-Doutora em Enfermagem. Professora do Programa de Mestrado Profissional em Ensino na Saúde/MPES, UFF, RJ, Brasil. Email: karinebrum@yahoo.com.br ORCID: https://orcid.org/0000-0002-1071-3587 


\section{Resumen}

Objetivos: Introducción: los datos de la Asociación Brasileña de Alergia e Inmunología indican que entre 6\% y $8 \%$ de los niños tienen alergias alimentarias, y entre ellos la alergia a la proteína de la leche de vaca (APLV) es la causa más común, actualmente considerada un problema de saludpública. Para los niños con reacciones alérgicas graves a los alimentos, el ambientefuera de la casa representa un alto riesgo de desencadenar anafilaxia. Analizando este escenario, se verifica la seriedad de tener una enfermera en la escuela. El objetivo de esta investigación es desarrollar una tecnología educativa en formato de Ebookpara enseñar la gestión de la seguridad de los estudiantes con APLV en la formación profesional de enfermeras y profesionales de la educación. Método: Estudio exploratorio descriptivo de enfoque cualitativo con desarrollo de investigación metodológica para la elaboración de una tecnología educativa. Los participantes serán estudiantes de enfermería de una Universidad Federal, profesionales de la educación de una escuela pública y privada en los municipios de Maricá y Niterói, respectivamente. La investigación obtuvo una evaluación positiva del Comité de Ética en Investigación de la Universidad Federal Fluminense. Se programarán tres talleres, donde se aplicará un cuestionario semiestructurado. El tratamiento y análisis de los datos se realizará de forma descriptiva, a través del análisis y clasificación del contenido. Resultados esperados: La intención és aportar beneficios a la enseñanza, la investigación y la sociedad, discutiendo temas que subsidiarán la reorientación del proceso de capacitación de las enfermeras, involucrándolos para que puedan actuar junto con los profesionales de la educación, a fin de alcanzar el objetivo principal, que es la calidad de cuidado y seguridad del estudiante con APLV.

Palabras clave: servicios de salud escolar, tecnología educativa, seguridad del paciente.

\section{Introdução}

O interesse pela temática partiu da experiência profissional da autora deste estudo, uma vez que como Tenente Enfermeira do Corpo de Bombeiros Militar do Estado do Rio de Janeiro trabalha com atendimento de emergência pré-hospitalar e acredita que pessoas informadas e treinadas, podem ajudar a salvar vidas. Somado a isso, na Pós-graduação em Controle de Infecção e Assistência à Saúde oferecida pela Escola de Enfermagem Aurora de Afonso Costa, a autora teve seu primeiro contato com a temática segurança do paciente, uma vez que seu trabalho de conclusão do curso foi um produto do Projeto Qualiseg UFF (atual LabQualiseg UFF).

Neste período, também conheceu as ações do Projeto Vida Sem Leite/Qualiseg UFF que discute as questões da alergia a proteína do leite de vaca na perspectiva da segurança do paciente.O LabQualiseg UFF trata-sedeumlaboratório de produçãode tecnologias educacionais de impacto na área de qualidade do cuidado e na segurança do paciente, como uma proposta na área de gerência em enfermagem do Departamento de Fundamentos de Enfermagem e Administração - MFE da Universidade Federal Fluminense (UFF). ${ }^{1}$

E para além da vida profissional, quando se tornou mãe, muitas questões a inquietaram no momento da escolha da escola para o seu filho, que possui alergias alimentares. Sendo esta uma das motivações que a levou a atuar como consultora e instrutora de primeiros socorros para ambiente escolar, onde identificou em sua práticaque há um déficit importante de conhecimento por parte dos educadores quando se trata dos assuntos relacionados à saúde do escolar, seja na prevenção de acidentes ou até mesmo na definição dos processos de trabalhos para o manejo de crianças com doenças ou condições crônicas, comopor exemplo a alergia a proteína do leite de vaca.

Segundo a Organização Mundial de Saúde (OMS),
$35 \%$ da população brasileira possui algum tipo de alergia. De acordo com o Ministério da Saúde, os principais tipos de alergia são as alergias alimentares. Estudos da Associação Brasileira de Alergia e Imunologia (ASBAI) afirmam que entre $6 \%$ e $8 \%$ das crianças e entre 2 e $3 \%$ dos adultos têm alergias alimentares (AA). E dentre todas, a alergia a proteína do leite de vaca (APLV) é a causa mais comum de alergia alimentar em crianças e adolescentes. $^{2}$

Atualmente as AA são consideradas um problema de saúde pública, pois a sua prevalência tem aumentado no mundo todo. ${ }^{2}$ Nos Estados Unidos, por exemplo, esta condição crônica é a principal causa de anafilaxia tratada em serviços de emergência, especialmente na população mais jovem. ${ }^{3}$ E para criança que apresenta reações alérgicas graves o ambiente extradomiciliar (como escolas, creches, festas, praças de alimentação) representa alto risco para desencadear anafilaxia e outras situações que ameacem a vida. ${ }^{2}$ É na escola que a criança passa boa parte do seu dia, e, em muitos casos, até a maior parte dele, obtém cuidados de higiene, alimentação, estuda e às vezes recebe medicações. ${ }^{4}$

Ao analisar este cenário, constata-se a seriedade de se ter um profissional de saúde dentro da escola. ${ }^{4}$ No Brasil, nota-se que a maior parte das escolas públicas e privadas não possuem um profissional de saúde no seu organograma. ${ }^{5}$ Uma das preocupaçõesfrequentes dos pais e familiares de crianças alérgicas é sobre a atuação dos professores/auxiliares. ${ }^{2}$ Eles seriam capazes de reconhecer os sinais e sintomas de uma reação alérgica grave ou choque anafilático apresentados por seus filhos e atuar nesta emergência? Saberiam identificar os riscos nas atividades do dia-a-dia, para que possam planejar e incluir a criança alérgica?

Sabe-se que o enfermeiro pela essência de sua formação é comprometido com a saúde, a qualidade de vida, com os familiares e a coletividade. Este profissional é imprescindível para o desenvolvimento integral da criança, aliado aos profissionais de educação, envolve- 
se na construção de um ambiente com o menor risco possível de adoecimento e de acidentes, associandose ao cuidar, prevenir, educar, visando à promoção de saúde destes indivíduos, que se encontram em fase de grande vulnerabilidade. ${ }^{6,7}$

Neste intuito, este estudo terá como produto a construção de um Ebook como uma tecnologia educacional para o ensino de enfermeiros sobre como gerenciar o cuidado seguro da criança com risco de episódios de alergias e reações anafiláticas dentro do ambiente escolar, subsidiando a tomada de decisão para atuarem preventivamente, o que atualmente representa um desafio para todos os segmentos, com propósito de minimizar os impactos na saúde, objetivando a redução do número de acidentes escolares, hospitalizações desnecessárias e até danos maiores ao indivíduo.

Com tantas possibilidades de informações que podem garantir a segurança da criança alérgica na escola, esta Pesquisa quer alcançar na área de enfermagem os estudantes de graduação e pósgraduação, profissionais, docentes, se estendendo aos educadores, familiares e alérgicos através da produção de uma tecnologia educacional digital que possa levar informação, utilizando os enfermeiros em formação como multiplicadores de conhecimento e para corroborar com este desafio do planejamento conjunto de ações de segurança da criança comAPLV no ambiente escolar.

Questão de pesquisa: De que forma as tecnologias educacionais no ensino de estudantes de enfermagem sobre o gerenciamento em segurança e sua atuação nas escolas poderiam contribuir no processo ensinoaprendizagem para identificação dos riscos e prevenção de eventos adversos no cuidado da criança com alergia a proteína do leite de vaca?

Objetivos pretendidos: $\mathrm{O}$ estudo tem como objetivo geraldesenvolver tecnologia educacional no formato de Ebook como estratégia inovadora para o ensino do gerenciamento em segurança do escolar com alergia a proteína do leite de vaca na formação profissional dos enfermeiros e para os profissionais de educação. E como objetivos específicos: 1) Analisar as publicações nacionais e internacionais existentes sobre gerenciamento da segurança do escolar alérgico a proteína do leite de vaca com risco de anafilaxia e sobre tecnologias educacionais para o ensino do manejo da anafilaxia no ambiente escolar; 2) Identificar o conhecimento dos graduandos de enfermagem do último ano de graduação e dos profissionais de educação sobre o gerenciamento de segurança do escolar APLV com risco de anafilaxia; 3) Elaborar um Plano de Intervenção para o gerenciamento seguro do escolar APLV utilizando oficinas como estratégia de ensino-aprendizagem para os enfermeiros em sua formação profissional e para os profissionais de educação sobre o gerenciamento da segurança do escolar APLV; 4) Produzir um Ebook como ferramenta pedagógica a partir dos resultados obtidos nas oficinas; 5) Propor um plano de inserção do produto na prática do ensino em saúde na formação profissional; 6) Validar o Ebook através de um painel de experts.

\section{Método}

Trata-se de um estudo descritivo e exploratório, de abordagem qualitativa com desenvolvimento de pesquisa metodológica para elaboração e validação de tecnologias educacionais para o ensino em saúdesobre o gerenciamento da segurança do escolar com APLV, utilizando oficinas como ferramenta metodológica de pesquisa para coleta de dados e como estratégia de ensino-aprendizagem para os enfermeiros em sua formação profissional e também para os profissionais de educação. ${ }^{8,9}$

A abordagem prioritária será a qualitativa, pois busca evidenciar os aspectos dinâmicos e holísticos da experiência humana ${ }^{7}$. A coleta de dados se dará durante a oficina, utilizando como instrumento um questionáriosemiestruturadodo Google Forms ${ }^{\circledR}$, autoaplicável, composto por questões fechadas e abertas, abordando a temática referente à segurança da criança alérgica a leite no ambiente escolar. ${ }^{9}$

Utilizar o espaço da oficina como estratégia metodológica de pesquisa destaca a articulação entre duas dimensões primordiais e indissociáveis da construção de conhecimento sobre articulações teórico-metodológicas e implicações ético políticas, com a riqueza da reflexão em grupo, promovendo um ambiente de trocas que possibilitam a discussão sobre a temática proposta gerando discordâncias construtivas com vistas ao envolvimento político de transformação. Ou seja, não se limita a coleta de dados, mas provoca a sensibilização dos participantes sobre a pluralidade do tema e a oportunização de conhecer diversos pontos de vista para determinado assunto 9 .

A pesquisa será realizada em três cenários distintos. O primeiro será em uma Universidade Pública na cidade de Niterói, entre outubro e dezembro de 2019. Participarão como população do estudo 15 alunos regularmente matriculados no curso de graduação em enfermagem do último ano ( $8^{\circ}$ e $9^{\circ}$ período $)$, pois deseja-se medir o conhecimento que estes profissionais de saúde estão recebendo na sua formação, pois logo mais serão lançados no mercado de trabalho.

O segundo cenário será composto por uma escola pública de educação infantil, e o terceiro por uma instituição privada de ensino, onde serão feitas duas oficinas complementares com 15 participantes cada, que irão corroborar com a pesquisa na construção do Ebook a partir do ponto de vista dos profissionais de educação. A escola particular participará mediante carta de autorização da instituição participante assinada pela 
direção. Já a escola municipal, colaborará com a pesquisa através de autorização da secretaria de educação do seu município.

A pesquisa obteve parecer favorável pelo Comitê de Ética em Pesquisa da Universidade Federal Fluminense (CAAE: 14347419.8.0000.5243).A participação na pesquisa será voluntária, os participantes serão convidados a participar da oficina e assinarão o Termo de Consentimento Livre e Esclarecido conforme Resoluções $n^{\circ}$ 466/12, 510/2016 e 580/2018 do Conselho Nacional de Saúde (CNS).

Os resultados das questões fechadas serão analisados em frequência e porcentagem através do Google Forms ${ }^{\circledR}$. Já os resultados das questões abertas serão analisados de forma descritiva, através da análise e classificação do conteúdo das respostas, conforme proposto por Minayo?.

A próxima etapa consistirá então, na construção do ebook a partir das considerações do público-alvo, compilando conteúdos sobre as ações de segurança, Plano de Prevenção de Riscos na escola, ferramentas para o ensino da segurança do escolar e gerenciamento do cuidado.

A escolha de um livro digital (ebook) se deu por conta do baixo custo para disponibilização, não existem custos para impressão, além de utilizar um pequeno espaço de memória e é compatível com as principais tecnologias móveis utilizadas atualmente, como telefones celulares, tablets e em notebooks. ${ }^{10}$

Será elaborado também para compor o Ebook um Plano de Prevenção de Riscos para o gerenciamento de segurança do escolar alérgico APLV e um Mapa de Riscos alertando os ambientes mais suscetíveis de incidentes e eventos adversos, utilizando os dados obtidos das oficinas que serão agrupados e consolidados por análise temática e discutidos à luz da fundamentação teórica e Políticas Públicas existentes.

Posteriormente, o Ebook será submetido à avaliação por experts na área de segurança do paciente, saúde do escolar e ensino na saúde..$^{11} \mathrm{~A}$ busca por estes especialistas será realizada na plataforma Lattes, que é a base de dados de currículos e instituições das áreas de Ciência e Tecnologia do Conselho Nacional de Desenvolvimento Científico e Tecnológico (CNPq).

Ao final da avaliação, pelos especialistas e considerando o percentual de concordância de cada item pelos experts será obtida a versão final do Ebook que será hospedado no endereço eletrônico do Projeto de Extensão LabQualiSEG UFF para download gratuito.

\section{Resultados esperados}

Este estudo pretende trazer benefícios ao ensino, a pesquisa, a assistência e a sociedade, a partir do momento em que se discutirá questões que subsidiarão a reorientação do processo de formação dos profissionais enfermeiros, bem como os processos de trabalho mediante a práxis de profissionais que atuam nas escolas, a fim de contribuir para prevenção de eventos adversos e incidentes relacionados à assistência do escolar com APLV, orientando-os nas ações coletivas do cotidiano escolar de forma inclusiva, a fim de alcançar a principal meta que é a qualidade do cuidado e a segurança do escolar com alergia alimentar.

\section{Referências}

1. UFF. Projeto Qualiseg: a qualidade em saúde e a segurança do paciente da gestão de risco. Disponível em: http://www.extensao.uff.br/?q=content/ projeto-qualiseg-qualidade-em-sa $\% \mathrm{C} 3 \%$ BAde-e-seguran $\% \mathrm{C} 3 \% \mathrm{~A} 7 \mathrm{a}$-dopaciente-na-gest $\%$ C3\%A3o-de-risco Acessado em 15/06/2018

2. Solé D, Silva LR, Cocco RR, Ferreira CT, Sarni RO, Oliveira LC et all Consenso Brasileiro sobre alergia alimentar: 2018 - Parte 2 - Diagnóstico, tratamento e prevenção. Documento Conjunto elaborado pela Sociedade Brasileira de Pediatria e Associação Brasileira de Alergia e Imunologia. Arq Asma AlergImunol. 2018; 2(1):39-82.

3. Benjamin LW. Anaphylaxis and Epinephrine in North Carolina Public Schools. Ann AllergyAsthmanImmunol. 2015 july; 115 (1): $75-77$ doi: 10.1016/j.anai.2015.04.008.

4. Valério M, Farias RM. A importância do profissional enfermeiro em uma instituição de educação infantil: Um estudo de caso sobre suas atribuições - Escola Municipal de Ensino Fundamental Senador Darcy Ribeiro - Porto Velho-RO,2015. Disponível em:http://repositorio.saolucas.edu.br:8080 xmlui/bitstream/handle/123456789/1559/tcc\%20margo\%20e\%20milla\%20 formatado $\% 2014122015$.pdf? sequence $=1 \&$ is Allowed $=y$

5. Simpósio Ibero-americano de Cooperação para o desenvolvimento e a Integração Regional, VIII., 2017, Rio Grande Do Sul. A Escola e seus Atores: os funcionários de escola. GT V - Desenvolvimento e Novas Tecnologias Produtivas e Sociais. São Pedro: UFFS, 2017. 16 f.2 v. Disponível em: $<$ https://www.uffs.edu.br/campi/cerro-largo/repositorio-ccl/anais-viiisimposio-iberoamericano-de-cooperacao-para-o-desenvolvimento-e-aintegracao-regional/a-escola-e-seus-atores-os-funcionarios-de-escola/@@ download/file>. Acesso em: 05 out. 2019.

6. Brasil. Ministério da Saúde. Saúde na escola. Brasília: Ministério da Saúde, 2009. (Cadernos de Atenção Básica, n. 24) (Série A. Normas e Manuais Técnicos). Disponível em: <http://189.28.128.100/dab/docs/publicacoes/ cadernos_ab/abcad24.pdf $>$ Acessado em 05/10/2019.

7. SMSP, Secretaria da Saúde. Manual de prevenção de acidentes e primeiros socorros nas escolas. Secretaria da Saúde. Coordenação de Desenvolvimento de Programas de Saúde. CODEPPS. São Paulo: SMS, 2007. 129p. Acesso em: 26/09/2019. Disponivel em: <https://www.amavi.org.br/arquivo/colegiados/ codime/2016/Primeiros_Socorros Manual Prev_Acid Escolas.pdf $>$

8. SpinkMJ,Menegon VM, Medrado B. Oficinas como estratégia de pesquisa: articulações teórico-metodológicas e aplicações ético-políticas. Psicologia \& Sociedade,2014 26(1)32-43. Disponível em: , http://www.scielo.br/pdf/psoc/ v26n1/05.pdf>Acesso em: 05/10/2019.

9. Minayo MCS. O desafio do conhecimento: pesquisa qualitativa em saúde Ed. 14, Săo Paulo: Hucitec, 2014.

10. Padilha ASC. Criando materiais digitais interativos : livros digitais e infográficos . Revista Tecnologias na Educação- Ano

8.Número/Vol.15-Edição Temática-TICs na EscolaAgosto2016tecnologiasnaeducacao.pro.br. Disponível em: $<$ http://tecedu.pro.br/wpcontent/uploads/2016/08/Texto5-Criando-materiais-digitais-interativo.pdf> Acesso em: 05/10/2019.

11. Teixeira E, Mota VMSS, organizadoras. Tecnologias educacionais em foco. São Caetano do Sul (SP): Difusão; 2011. 